\title{
Cardiovascular Risk Factors and the Severity of COVID-19 Disease
}

Imane Motaib ${ }^{1}$, Saad Zbiri ${ }^{2}$, Saloua Elamari ${ }^{3}$, Amal Haoudar ${ }^{4}$, Asma Chadli ${ }^{3}$, Chafik El Kettani ${ }^{4}$

1. Endocrinology, Diabetes, and Metabolism, Mohammed VI University of Health Sciences (UM6SS), Casablanca, MAR

2. Epidemiology and Public Health, Laboratory of Public Health, Health Economics, and Health Management, International School of Public Health, Mohammed VI University of Health Sciences (UM6SS), Casablanca, MAR 3. Endocrinology, Diabetology, Metabolic Disease, and Nutrition, Mohammed VI University of Health Sciences (UM6SS), Casablanca, MAR 4. Anesthesia and Critical Care, Cheikh Khalifa International University Hospital, Mohammed VI University of Health Sciences (UM6SS), Casablanca, MAR

Corresponding author: Imane Motaib, imanemotaib@gmail.com

\section{Abstract \\ Background and objective}

Several cardiovascular risk factors have emerged as important determinants of severe illness and death among coronavirus disease 2019 (COVID-19) patients. However, the full impact of these cardiovascular risk factors is still under investigation. This study aimed to investigate the association between patients' level of cardiovascular risk and the severity of COVID-19.

\section{Materials and methods}

This observational study included all adult patients with COVID-19 hospitalized at Sheikh Khalifa Ibn Zaid International University Hospital from March 20 to May 10, 2020. The cardiovascular risk level was assessed by the doctor responsible for each patient based on the 2019 European Society of Cardiology (ESC), the European Atherosclerosis Society (EAS), and the European Association for the Study of Diabetes (EASD) guidelines. We examined the association between the patients' level of cardiovascular risk and their severity of COVID-19 disease by using a logistic regression model.

\section{Results}

Among 133 patients with confirmed COVID-19, 46.6\% had a low cardiovascular risk level, 19.5\% had a moderate risk level, $15.8 \%$ had a high risk level, and $18.1 \%$ was found to have a very high risk level. Patients with different cardiovascular risk levels had significantly different rates of complications including secondary infection ( $p$-value: $<0.001$ ), acute respiratory distress syndrome (ARDS) $(p$-value $=0.017$ ), intensive care unit (ICU) admission ( $\mathrm{p}$-value: $<0.001$ ), and death ( $\mathrm{p}$-value: $<0.001)$. A patient's very high cardiovascular risk level versus low, moderate, or high cardiovascular risk level was independently associated with ICU admission [OR $=6.42,95 \%$ CI: $(1.45-28.30)]$.

Review began 02/12/2021 Review ended 05/28/2021 Published 06/07/2021

\section{() Copyright 2021}

Motaib et al. This is an open access article distributed under the terms of the Creative Commons Attribution License CC-BY 4.0., which permits unrestricted use, distribution, and reproduction in any medium, provided the original author and source are credited.

\section{Conclusion}

Based on our findings, an increased level of cardiovascular risk among patients was strongly associated with the severity of COVID-19. This study also highlights the need for assessing cardiovascular risk factors in all patients with COVID-19.

Categories: Cardiology, Endocrinology/Diabetes/Metabolism, Epidemiology/Public Health Keywords: traditional cardiovascular risk factors, covid 19, severity markers, sar-cov 2 infection

\section{Introduction}

The novel coronavirus disease 2019 (COVID-19) was first identified in Wuhan, China in December 2019, and since then it has spread rapidly around the world. The World Health Organization (WHO) subsequently declared COVID-19 as a global pandemic on March 11, 2020 [1]. As of April 14, 2021, there have been $136,996,364$ confirmed cases of COVID-19 globally, which have resulted in 2,951,832 deaths [2].

Previous studies on the condition have observed that elderly patients and those with comorbidities such as diabetes, hypertension, and cardiovascular diseases are more likely to develop severe forms of COVID-19, with more complications and/or more susceptibility to death from the disease [3,4]. However, the impact of these cardiovascular risk factors such as age, hypertension, diabetes, and cardiovascular diseases on the clinical outcomes and the prognosis of COVID-19 disease is still poorly understood.

This study investigated the association between the level of the patients' cardiovascular risk and the severity of COVID-19. We hypothesized that patients with increased cardiovascular risk levels, compared to those 


\section{Materials And Methods}

\section{Study design}

This single-center observational study included all adult patients (aged 18 years and above) admitted with confirmed COVID-19 disease to the Sheikh Khalifa Ibn Zaid International University Hospital in Casablanca, Morocco, between March 20 and May 10, 2020. This hospital has been mandated by the Moroccan Ministry of Health to care for patients with COVID-19. Depending on the severity of COVID-19, patients were admitted to the intensive care unit (ICU) (severe patients) or provided non-ICU care (non-severe patients). Criteria of ICU admission were defined based on the WHO interim guidelines: respiratory rate of $>30$ breaths per minute, severe respiratory distress or oxygen saturation level of $\mathrm{SpO}_{2}$ below $92 \%$ with 41 of oxygen

ventilation, or neurological or hemodynamic disorders [5]. Pregnant women and patients under the age of 18 were excluded from the study.

\section{Data collection}

Data were collected from the electronic medical records of patients by a trained team of physicians. These medical investigators gathered and reviewed the data of all patients.

For each patient, we collected data related to her/his cardiovascular risk level as well as demographic characteristics including age and gender; comorbidities including hypertension, diabetes, cardiovascular diseases (atherosclerotic cardiovascular disease, heart failure, arrhythmia, etc.), respiratory diseases (chronic obstructive pulmonary disease, asthma, etc.), dyslipidemia, and other diseases and risk factors (hyperuricemia, neoplasia, smoking, obesity, etc.); clinical symptoms including fever, general symptoms (dizziness, myalgias, asthenia, etc.), respiratory symptoms (dyspnea, cough, etc.), as well as ear, nose, and throat (ENT) and digestive symptoms; and clinical outcomes including intensive care unit (ICU) admission, invasive mechanical ventilation use, the onset of complications [acute respiratory distress syndrome (ARDS), secondary infection, multiple organ failure, thromboembolic disease], and deaths.

\section{Outcomes definition}

The diagnosis of COVID-19 was based on the WHO interim guidelines [6] and confirmed as per reverse transcription-polymerase chain reaction (RT-PCR) assays. Diabetes was defined as a self-reported medical history of diabetes. Newly diagnosed diabetes was defined based on the American Diabetes Association (ADA) criteria [7], including fasting plasma glucose of over $126 \mathrm{mg} / \mathrm{dl}$ and/or random glycemia higher than $200 \mathrm{mg} / \mathrm{dl}$ and classic signs of hyperglycemia and/or HbA1c of $\geqslant 6.5 \%$ during the hospital stay. Hypertension was defined as blood pressure above $140 / 90 \mathrm{mmHg}$, and ARDS was defined according to the Berlin definition [8].

\section{Outcomes assessment}

A cardiovascular risk assessment was performed for each patient by the doctor who had recommended her/his hospitalization. As shown in Table 1, doctors at the study hospital performed the cardiovascular risk stratification based on the 2019 European Society of Cardiology (ESC) and European Atherosclerosis Society (EAS) guidelines, and for patients with diabetes, as per the 2019 ESC and the European Association for the Study of Diabetes (EASD) guidelines $[9,10]$. This cardiovascular risk stratification is commonly used in many countries including Morocco [11]. Based on this, patients were classified into four cardiovascular risk level categories: very high risk level, high risk level, moderate risk level, and low risk level. 


\section{Cureus}

Risk

level

Very high

risk level

High risk

level

risk level

Low risk

level

\section{Description}

Documented ASCVD DM with target organ damage ${ }^{a}$, or at least three major risk factors, or early onset of T1DM of long duration (>20 years). Severe CKD (eGFR of $<30 \mathrm{~mL} / \mathrm{min} / 1.73 \mathrm{~m}^{2}$ ). A calculated SCORE of $>10 \%$ for 10 -year risk of fatal CVD. FH with ASCVD or another major risk factor

Markedly elevated single risk factors, in particular TC of $>8 \mathrm{mmol} / \mathrm{L}$ ( $>310 \mathrm{mg} / \mathrm{dL}$ ), LDL-C of $>4.9 \mathrm{mmol} / \mathrm{L}$ ( $>190 \mathrm{mg} / \mathrm{dL}$ ), or BP of $>180 / 110 \mathrm{mmHg}$. Patients with FH without other major risk factors. Patients with DM without target organ damage ${ }^{\mathrm{a}}$, with DM duration of $>10$ years, or another additional risk factor. Moderate CKD (eGFR of 30-59 mL/min/1.73 $\mathrm{m}^{2}$ ). A calculated SCORE of $>5 \%$ and $<10 \%$ for 10 -year risk of fatal CVD

Young patients (T1DM: $<35$ years; T2DM: $<50$ years) with DM duration of $<10$ years, without other risk factors. Calculated SCORE of $>1 \%$ and $<5 \%$ for 10 -year risk of fatal CVD

Calculated SCORE of $<1 \%$ for 10 -year risk of fatal CVD

\section{TABLE 1: Cardiovascular risk level categories*}

${ }^{*}[9,10]$

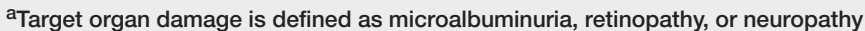

ASCVD: atherosclerotic cardiovascular disease; BP: blood pressure; CKD: chronic kidney disease; CVD: cardiovascular disease; DM: diabetes mellitus; eGFR: estimated glomerular filtration rate; FH: familial hypercholesterolemia; LDL-C: low-density lipoprotein cholesterol; SCORE:

Systematic Coronary Risk Estimation; T1DM: type 1 DM; T2DM: type 2 DM; TC: total cholesterol

\section{Ethical considerations}

The study was approved by the Institutional Ethics Board of the Sheikh Khalifa Ibn Zaid International University Hospital (approval number: CE_UM6SS/1/06/2020 - April 3, 2020). Patient consent was waived as the study included only unidentifiable data, in accordance with the national law.

\section{Statistical analysis}

For descriptive analysis, we presented continuous variables as medians with interquartile ranges (IQRs) (small sample) and categorical variables as percentages. Patients with different cardiovascular risk level categories were compared in terms of demographics characteristics, comorbidities, clinical symptoms, and clinical outcomes, using the nonparametric k-sample test on the equality of medians for continuous variables and using the Fisher's exact test for categorical variables.

Secondly, univariate analysis was performed to study the association between all available variables, as described above, and ICU admissions. Finally, multivariate logistic regression was performed to examine the association between cardiovascular risk level and ICU admissions. Since cardiovascular risk might be highly correlated with many variables, particularly those related to comorbidities, we used a stepwise multivariate model based on a bidirectional elimination, with a $p$-value of $<0.05$ and including all significant control variables as found in the univariate analysis. Results were reported as odds ratios (ORs) with their $95 \%$ confidence intervals (CIs).

All statistical analyses were performed using the STATA software (StataCorp LLC, College Station, TX). Pvalues were two-sided, and those $<0.05$ were considered statistically significant.

\section{Results}

A total of 133 adult patients who were hospitalized at Sheikh Khalifa Ibn Zaid International University Hospital with confirmed COVID-19 infection from March 20 to May 10, 2020, were included in the study. Table 2 presents the characteristics of the study population. The median age of included patients was 53 years (IQR: 36-64 years); 55.6\% of the included patients were male. The most common cardiovascular risk factors were hypertension (28.6\%), diabetes (14.3\%), and preexisting cardiovascular disease (13.5\%). Among the study population, $46.6 \%$ had a low cardiovascular risk level, $19.5 \%$ had a moderate cardiovascular risk level, $15.8 \%$ had a high cardiovascular risk level, and $18.1 \%$ had a very high cardiovascular risk level. 


\section{Cureus}

Variables

Values

Demographics

Age, years, median (IQR)

53 (36-64)

Male gender, n (\%)

Cardiovascular risk, n (\%)

Low level

Moderate level

High level

Very high level

Comorbidities, n (\%)

Hypertension

Diabetes

Cardiovascular disease

Respiratory disease

Dyslipidemia

Other diseases $^{\mathrm{a}}$

Clinical symptoms, n (\%)

Fever

General symptoms ${ }^{b}$

Respiratory symptoms

ENT symptoms

Digestive symptoms

32 (24.1)

Outcomes, n (\%)

Secondary infection

Invasive mechanical ventilation

ARDS

$13(9.8)$

Thromboembolic complication

4 (3)

Multi-organ failure

$9(6.8)$

ICU admission

46 (34.6)

Death

\section{TABLE 2: Characteristics of the study population}

aDizziness, myalgias, asthenia, etc.

bHyperuricemia, neoplasia, smoking, obesity, etc.

ENT: ear, nose, and throat; ARDS: acute respiratory distress syndrome; ICU: intensive care unit; IQR: interquartile range

The characteristics of the study population as per their cardiovascular risk levels are presented in Table 3. Patients with different cardiovascular risk levels were comparable in terms of clinical symptoms (p-value: $>0.05$ ), but significantly differed in terms of demographics including age ( $\mathrm{p}$-value: $<0.001)$ and gender $(\mathrm{p}-$ value $=0.028$ ), in terms of comorbidities including hypertension ( $p$-value: $<0.001)$, diabetes ( $p$-value: 
$<0.001$ ), cardiovascular disease ( $\mathrm{p}$-value: $<0.001$ ), dyslipidemia ( $\mathrm{p}$-value: $=0.008$ ), and other diseases ( $\mathrm{p}$ value $=0.041)$, and in terms of clinical outcomes including secondary infection ( $p$-value: $<0.001)$, requirement for invasive mechanical ventilation ( $p$-value $=0.001$ ), ARDS ( $p$-value $=0.017$ ), ICU admission ( $\mathrm{p}$-value: $<0.001$ ), and death ( $\mathrm{p}$-value: $<0.001)$. However, there were no significant differences regarding thromboembolic complications and multi-organ failure (p-values of 0.743 and 0.148 , respectively). Notably, patients with a very high cardiovascular risk level also had higher rates of secondary infections, invasive mechanical ventilations, ARDS, ICU admissions, and deaths, when compared to patients with lower levels of cardiovascular risk (low, moderate, or high level of cardiovascular risk).

\begin{tabular}{|c|c|c|c|c|c|}
\hline Variables & $\begin{array}{l}\text { Low risk level }(n= \\
62)\end{array}$ & $\begin{array}{l}\text { Moderate risk level }(n= \\
\text { 26) }\end{array}$ & $\begin{array}{l}\text { High risk level }(n= \\
\text { 21) }\end{array}$ & $\begin{array}{l}\text { Very high risk level }(n= \\
\text { 24) }\end{array}$ & P-value \\
\hline \multicolumn{6}{|l|}{ Demographics } \\
\hline Age, years, median (IQR) & $36(26-46)$ & $58(54-64)$ & 66 (59-73) & $72(63-76)$ & $<0.0001$ \\
\hline Male, $\mathrm{n}(\%)$ & $26(41.9)$ & $18(69.2)$ & $15(71.4)$ & $15(62.5)$ & 0.028 \\
\hline \multicolumn{6}{|l|}{ Comorbidities, n (\%) } \\
\hline Hypertension & $0(0)$ & $8(30.8)$ & $12(57.1)$ & $18(75)$ & $<0.0001$ \\
\hline Diabetes & $0(0)$ & $0(0)$ & $8(38.1)$ & $11(45.8)$ & $<0.0001$ \\
\hline Cardiovascular diseases & $0(0)$ & $0(0)$ & $1(4.8)$ & $17(70.8)$ & $<0.0001$ \\
\hline Respiratory diseases & $5(8.1)$ & $2(7.7)$ & $3(14.3)$ & $1(4.2)$ & 0.672 \\
\hline Dyslipidemia & $3(4.8)$ & $0(0)$ & $2(9.5)$ & $6(25)$ & 0.008 \\
\hline Other diseases ${ }^{\mathrm{a}}$ & $4(6.5)$ & $5(19.2)$ & $1(4.8)$ & $6(25)$ & 0.041 \\
\hline \multicolumn{6}{|l|}{ Clinical symptoms, n (\%) } \\
\hline Fever & $22(35.5)$ & $13(50)$ & $13(61.9)$ & $12(50)$ & 0.160 \\
\hline General symptoms ${ }^{b}$ & $23(37.1)$ & $10(38.5)$ & $9(42.9)$ & $13(54.2)$ & 0.536 \\
\hline Respiratory symptoms & $30(48.4)$ & $15(57.7)$ & $15(71.4)$ & $17(70.8)$ & 0.143 \\
\hline ENT symptoms & $21(33.9)$ & $7(26.9)$ & $6(28.6)$ & $6(25)$ & 0.868 \\
\hline Digestive symptoms & $15(24.2)$ & $6(23.1)$ & $7(33.3)$ & $4(16.7)$ & 0.636 \\
\hline \multicolumn{6}{|l|}{ Outcomes, n (\%) } \\
\hline Secondary infection & $3(4.8)$ & $6(23.1)$ & $6(28.6)$ & $12(50)$ & $<0.0001$ \\
\hline $\begin{array}{l}\text { Invasive mechanical } \\
\text { ventilation }\end{array}$ & $1(1.6)$ & $4(15.4)$ & $2(9.5)$ & $7(29.2)$ & 0.001 \\
\hline ARDS & $2(3.2)$ & $3(11.5)$ & $2(9.5)$ & $6(25)$ & 0.017 \\
\hline complications & $2(3.2)$ & $0(0)$ & $1(4.8)$ & $1(4.2)$ & 0.743 \\
\hline Multi-organ failure & $2(3.2)$ & $2(7.7)$ & $1(4.8)$ & $4(16.7)$ & 0.148 \\
\hline ICU admissions & $6(9.7)$ & $9(34.6)$ & $11(52.4)$ & $20(83.3)$ & $<0.0001$ \\
\hline Death & $1(1.6)$ & $2(7.7)$ & $2(9.5)$ & $9(37.5)$ & $<0.0001$ \\
\hline
\end{tabular}

\section{TABLE 3: Characteristics of the study population according to their cardiovascular risk level}

aDizziness, myalgias, asthenia, etc

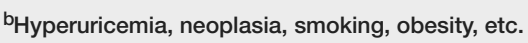

ENT: ear, nose, and throat; ARDS: acute respiratory distress syndrome; ICU: intensive care unit; IQR: interquartile range 


\section{Cureus}

For the univariate analysis (Table 4), we found that the cardiovascular risk level was associated with ICU admission. In fact, compared to patients with a low level of cardiovascular risk, only those patients with a very high level of cardiovascular risk were more likely to require ICU admission [OR = 46.67, 95\% CI: (11.93182.60)]. Furthermore, patients with a very high cardiovascular risk level were still more likely to require ICU admission when compared with all other patients including those with low, moderate, or high levels of cardiovascular risk [OR $=15.96,95 \% \mathrm{CI}:(5.00-50.94)]$.

For the multivariate analysis (Table 4), we found that patients having very high cardiovascular risk levels were independently associated with ICU admission. Indeed, these patients with a very high cardiovascular risk level were more likely to require ICU admission when compared with patients with low cardiovascular risk level [OR = 11.94, 95\% CI: $(1.34-106.78)]$ and also when compared with all other patients including those with a low, moderate, or high levels of cardiovascular risk [OR $=6.42,95 \% \mathrm{CI}$ : (1.45-28.30)]. 


\section{Cureus}

\begin{tabular}{|c|c|c|c|}
\hline Variables & Univariate model, OR (95\% CI) & Multivariate model ${ }^{\mathrm{a}}$, OR $(95 \% \mathrm{Cl})$ & Multivariate model ${ }^{\mathrm{b}}$, OR $(95 \% \mathrm{Cl})$ \\
\hline \multicolumn{4}{|l|}{ Demographics } \\
\hline Age, years & $1.08(1.05-1.11)$ & $1.05(1.00-1.11)$ & $1.07(1.03-1.11)$ \\
\hline Male & $3.92(1.76-8.70)$ & $3.19(1.05-9.69)$ & $3.24(1.07-9.81)$ \\
\hline \multicolumn{4}{|l|}{ Cardiovascular risk } \\
\hline Low level & 1 & 1 & \\
\hline Moderate level & $4.94(1.54-15.87)$ & $1.64(0.34-7.98)$ & \\
\hline High level & $10.27(3.09-34.12)$ & $2.17(0.32-14.68)$ & \\
\hline Very high level & $46.67(11.93-182.60)$ & $11.94(1.34-106.78)$ & \\
\hline \multicolumn{4}{|c|}{ Very high cardiovascular risk level } \\
\hline No & 1 & 1 & \\
\hline Yes & $15.96(5.00-50.94)$ & & $6.42(1.45-28.30)$ \\
\hline \multicolumn{4}{|l|}{ Comorbidities } \\
\hline Hypertension & $4.80(2.15-10.71)$ & & \\
\hline Diabetes & $4.03(1.46-11.13)$ & & \\
\hline Cardiovascular diseases & $13.55(3.67-50.02)$ & & \\
\hline Respiratory diseases & $2.46(0.71-8.55)$ & & \\
\hline Dyslipidemia & $1.65(0.47-5.72)$ & & \\
\hline Other diseases $^{c}$ & $3.75(1.27-11.10)$ & $5.95(1.20-29.41)$ & $6.55(1.33-32.19)$ \\
\hline \multicolumn{4}{|l|}{ Clinical symptoms } \\
\hline Fever & $2.33(1.12-4.82)$ & & \\
\hline General symptoms $^{\mathrm{d}}$ & $1.97(0.95-4.07)$ & & \\
\hline Respiratory symptoms & $4.83(2.08-11.21)$ & $6.24(1.96-19.84)$ & $6.51(2.06-20.58)$ \\
\hline ENT symptoms & $1.03(0.47-2.23)$ & & \\
\hline Digestive symptoms & $1.18(0.52-2.70)$ & & \\
\hline
\end{tabular}

\section{TABLE 4: Association of cardiovascular risk with ICU admission}

aln this model, we included the patient's "cardiovascular risk" with four dummy variables (low level, moderate level, high level, and very high level)

bIn this model, we included the variable "very high cardiovascular risk level" only

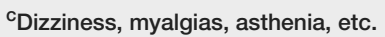

dHyperuricemia, neoplasia, smoking, obesity, etc.

ICU: intensive care unit; ENT: ear, nose, and throat; OR: odds ratio; Cl: confidence interval

\section{Discussion}

In this study, we investigated the association of cardiovascular risk levels of the patients with the severity of COVID-19 disease; this is the first major study involving an African population with COVID-19. The patients' level of cardiovascular risk was assessed by the doctor who had recommended their hospitalization, following the 2019 ESC, EAS, and EASD guidelines $[9,10]$.

Patients with a very high level of cardiovascular risk had a significantly higher rate of complications 
including secondary infection and ARDS. In addition, these patients also showed generally poorer clinical outcomes. More than four-fifths of them were admitted to the ICU and about one-third of them required invasive mechanical ventilation. The death rate was also higher among patients with a very high cardiovascular risk level. Interestingly, we found that a patient's very high cardiovascular risk level was strongly and independently associated with ICU admissions.

These findings are in agreement with previous reports that have linked worse outcomes in COVID-19 infection with cardiovascular risk factors such as advanced age, hypertension, and diabetes, as well as with cardiovascular diseases such as coronary heart disease, heart failure, and cerebrovascular infarction. Indeed, Wang et al. have reported higher rates of ICU admissions in patients with several comorbidities [12]. Furthermore, a recent prospective study involving an American cohort of 5,279 patients with COVID-19 has shown that advanced age followed by heart failure were the strongest risk factors for severe COVID-19 illness [13].

Similarly, a study that involved an Italian cohort of 1,591 infected patients with COVID-19 in the Lombardy region has shown that $68 \%$ of the patients admitted in ICU units were elderly individuals and had at least one comorbidity. The most common comorbidities in this study were hypertension (49\%) followed by cardiovascular disease (21\%) and hypercholesterolemia (18\%) [14].

Moreover, Guan et al. have reported that among 1,099 patients with laboratory-confirmed COVID-19 disease from 552 hospitals in 30 Chinese provinces, hypertension and diabetes were strongly associated with admission to the ICU unit, the use of mechanical ventilation, and death as a composite endpoint [15]. Likewise, the reported mortality rate was higher in patients with underlying cardiovascular diseases than patients without comorbidities [16]. WU et al. have reported that among patients who succumbed to the disease, $10.5 \%$ had cardiovascular disease, $7.3 \%$ had diabetes, and 6\% had hypertension [4]. Also, Li et al. have reported clinical characteristics of 25 death cases infected with COVID-19 pneumonia and found that among them, $64 \%$ had hypertension, $40 \%$ had diabetes, 32\% had heart diseases, $20 \%$ had kidney diseases, and $16 \%$ had cerebral infarction [17]. Similarly, an Italian study that described the characteristics of patients who died as a result of COVID-19 has observed that deceased patients were predominantly elderly males with multiple comorbidities [18].

Several mechanisms may be attributed to the association of cardiovascular risk factors with worse clinical outcomes in patients with COVID-19. Patients with a higher level of cardiovascular risk may have a lower grade of vascular inflammation, which combines with the immune response induced by the virus and leads to an aggravation of the inflammatory state [19]. Secondly, the procoagulant state reported in COVID-19 may increase the risk of thromboembolic and acute cardiovascular events, by activating prothrombotic factors in atheromatous plaque [20]. Finally, previous studies have reported a significant association of myocardial injury with fatal outcomes of COVID-19 [21]. Consequently, patients with cardiovascular risk factors are more likely to have a severe form of COVID-19.

\section{Limitations}

Our study has some limitations. Firstly, it was observational by design, and hence further multicentric prospective and/or retrospective studies are required to validate our results. Secondly, only 133 patients were included in the study, and a larger study including more participants is highly recommended in order to verify our results. Thirdly, our assessment of COVID-19 severity was based on ICU admissions, and factors related to disease progression were not analyzed extensively. Finally, this was a single-center study, and this may have led to some biases in the results. Therefore, further multicenter studies are recommended to rectify this issue.

\section{Conclusions}

This study found that an increased level of cardiovascular risk seems to be associated with the severity of COVID-19 disease. Further studies need to be conducted in order to study the mechanisms that induce and aggravate the severity of COVID-19 in patients with cardiovascular risk factors. Our findings also highlight the need for early assessment of cardiovascular risk levels in all patients with COVID-19, as well as implementing a priority vaccination strategy for patients with cardiovascular diseases.

\section{Additional Information \\ Disclosures}

Human subjects: Consent was obtained or waived by all participants in this study. Institutional Ethics Board of Cheikh Khalifa Ibn Zaid International University Hospital issued approval CE_UM6SS/1/06/2020 April 3, 2020. This study was approved by the Institutional Ethics Board of Cheikh Khalifa Ibn Zaid International University Hospital. Animal subjects: All authors have confirmed that this study did not involve animal subjects or tissue. Conflicts of interest: In compliance with the ICMJE uniform disclosure form, all authors declare the following: Payment/services info: All authors have declared that no financial support was received from any organization for the submitted work. Financial relationships: All authors have declared that they have no financial relationships at present or within the previous three years with 
any organizations that might have an interest in the submitted work. Other relationships: All authors have declared that there are no other relationships or activities that could appear to have influenced the submitted work.

\section{Acknowledgements}

Both authors (I.M and S.Z) have contributed equally to this article.

\section{References}

1. World Health Organization: rolling updates on coronavirus disease (COVID-19) . (2020). Accessed: March 31, 2020: https://www.who.int/emergencies/diseases/novel-coronavirus-2019/events-as-they-happen.

2. World Health Organization: Coronavirus Disease 2019 (COVID-19) Situation Report - 152 . (2020). Accessed: June 20, 2020: https://www.who.int/docs/default\%20source/coronaviruse/situation-reports/20200620covid-19-sitrep-152.pdf?sfvrsn=83af....

3. Zhou F, Yu T, Du R, et al.: Clinical course and risk factors for mortality of adult inpatients with COVID-19 in Wuhan, China: a retrospective cohort study. Lancet. 2020, 395:1054-62. 10.1016/S0140-6736(20)30566-3

4. Wu Z, McGoogan JM: Characteristics of and important lessons from the coronavirus disease 2019 (COVID19) outbreak in China: summary of a report of 72314 cases from the Chinese Center for Disease Control and Prevention. JAMA. 2020, 323:1239-42. 10.1001/jama.2020.2648

5. Peng F, Tu L, Yang Y, et al.: Management and treatment of COVID-19: the Chinese experience. Can J Cardiol. 2020, 36:915-30. 10.1016/j.cjca.2020.04.010

6. World Health Organization: clinical management of severe acute respiratory infection when novel coronavirus (nCoV) infection is suspected: interim guidance. (2020). Accessed: June 4, 2021: https://apps.who.int/iris/bitstream/handle/10665/330893/WHO-nCoV-Clinical-2020.3-eng.pdf? sequence $=1$ \&isAllowed $=\mathrm{y} \% 20$ (Ac....

7. American Diabetes Association: 2. Classification and diagnosis of diabetes: Standards of Medical Care in Diabetes-2020. Diabetes Care. 2020, 43:S14-31. 10.2337/dc20-S002

8. Ranieri VM, Rubenfeld GD, Thompson BT, et al.: Acute respiratory distress syndrome: the Berlin Definition . JAMA. 2012, 307:2526-33. 10.1001/jama.2012.5669

9. Mach F, Baigent C, Catapano AL, et al.: 2019 ESC/EAS Guidelines for the management of dyslipidaemias: lipid modification to reduce cardiovascular risk. Eur Heart J. 2020, 41:111-88. 10.1093/eurheartj/ehz455

10. Cosentino F, Grant PJ, Aboyans V, et al.: 2019 ESC Guidelines on diabetes, pre-diabetes, and cardiovascular diseases developed in collaboration with the EASD. Eur Heart J. 2020, 41:255-323. 10.1093/eurheartj/ehz486

11. Delahaye F: Recommendations of the European Society of Cardiology on cardiovascular prevention . Réalités Cardiologiques. 2017, 20:1-5.

12. Wang D, Hu B, Hu C, et al.: Clinical characteristics of 138 hospitalized patients with 2019 novel coronavirus-infected pneumonia in Wuhan, China. JAMA. 2020, 323:1061-9. 10.1001/jama.2020.1585

13. Petrilli CM, Jones SA, Yang J, et al.: Factors associated with hospital admission and critical illness among 5279 people with coronavirus disease 2019 in New York City: prospective cohort study. BMJ. 2020, 369:m1966. 10.1136/bmj.m1966

14. Grasselli G, Zangrillo A, Zanella A, et al.: Baseline characteristics and outcomes of 1591 patients infected with SARS-CoV-2 admitted to ICUs of the Lombardy Region, Italy. JAMA. 2020, 323:1574-81. 10.1001/jama.2020.5394

15. Guan WJ, Ni ZY, Hu Y, et al.: Clinical characteristics of coronavirus disease 2019 in China . N Engl J Med. 2020, 382:1708-20. 10.1056/NEJMoa2002032

16. Epidemiology Working Group for NCIP Epidemic Response, Chinese Center for Disease Control and Prevention: The epidemiological characteristics of an outbreak of 2019 novel coronavirus diseases (COVID19) in China (Article in Chinese). Zhonghua Liu Xing Bing Xue Za Zhi. 2020, 41:145-51. 10.3760/cma.j.issn.0254-6450.2020.02.003

17. Li X, Wang L, Yan S, et al.: Clinical characteristics of 25 death cases with COVID-19: a retrospective review of medical records in a single medical center, Wuhan, China. Int J Infect Dis. 2020, 94:128-32. 10.1016/j.ijid.2020.03.053

18. Onder G, Rezza G, Brusaferro S: Case-fatality rate and characteristics of patients dying in relation to COVID-19 in Italy. JAMA. 2020, 323:1775-6. 10.1001/jama.2020.4683

19. Savoia C, Schiffrin EL: Vascular inflammation in hypertension and diabetes: molecular mechanisms and therapeutic interventions. Clin Sci (Lond). 2007, 112:375-84. 10.1042/CS20060247

20. Wang J, Hajizadeh N, Moore EE, et al.: Tissue plasminogen activator (tPA) treatment for COVID-19 associated acute respiratory distress syndrome (ARDS): a case series. J Thromb Haemost. 2020, 18:1752-5. 10.1111/jth.14828

21. Ruan Q, Yang K, Wang W, Jiang L, Song J: Clinical predictors of mortality due to COVID-19 based on an analysis of data of 150 patients from Wuhan, China. Intensive Care Med. 2020, 46:846-8. 10.1007/s00134020-05991-x 